Algebraic $\&$ Geometric Topology

Volume 3 (2003) 777-789

Published: 24 August 2003

ATG

\title{
The Chess conjecture
}

\author{
RUSTAM SADYKOV
}

\begin{abstract}
We prove that the homotopy class of a Morin mapping $f: P^{p} \rightarrow$ $Q^{q}$ with $p-q$ odd contains a cusp mapping. This affirmatively solves a strengthened version of the Chess conjecture [5], [3]. Also, in view of the Saeki-Sakuma theorem [10] on the Hopf invariant one problem and Morin mappings, this implies that a manifold $P^{p}$ with odd Euler characteristic does not admit Morin mappings into $\mathbb{R}^{2 k+1}$ for $p \geq 2 k+1 \neq 1,3,7$.
\end{abstract}

AMS Classification 57R45; 58A20, 58K30

Keywords Singularities, cusps, fold mappings, jets

\section{Introduction}

Let $P$ and $Q$ be two smooth manifolds of dimensions $p$ and $q$ respectively and suppose that $p \geq q$. The singular points of a smooth mapping $f: P \rightarrow Q$ are the points of the manifold $P$ at which the rank of the differential $d f$ of the mapping $f$ is less than $q$. There is a natural stratification breaking the singular set into finitely many strata. We recall that the kernel rank $k r_{x}(f)$ of a smooth mapping $f$ at a point $x$ is the rank of the kernel of $d f$ at $x$. At the first stage of the stratification every stratum is indexed by a non-negative integer $i_{1}$ and defined as

$$
\Sigma^{i_{1}}(f)=\left\{x \in P \mid k r_{x}(f)=i_{1}\right\} .
$$

The further stratification proceeds by induction. Suppose that the stratum $\Sigma_{n-1}(f)=\Sigma^{i_{1}, \ldots, i_{n-1}}(f)$ is defined. Under assumption that $\Sigma_{n-1}(f)$ is a submanifold of $P$, we consider the restriction $f_{n-1}$ of the mapping $f$ to $\Sigma_{n-1}(f)$ and define

$$
\Sigma^{i_{1}, \ldots, i_{n}}(f)=\left\{x \in \Sigma_{n-1}(f) \mid k r_{x}\left(f_{n-1}\right)=i_{n}\right\} .
$$

Boardman [4] proved that every mapping $f$ can be approximated by a mapping for which every stratum $\Sigma_{n}(f)$ is a manifold.

We abbreviate the sequence $\left(i_{1}, \ldots, i_{n}\right)$ of $n$ non-negative integers by $I$. We say that a point of the manifold $P$ is an $I$-singular point of a mapping $f$ if 
it belongs to a singular submanifold $\Sigma^{I}(f)$. There is a class of in a sense the simplest singularities, which are called Morin. Let $I_{1}$ denote the sequence $(p-q+1,0)$ and for every integer $k>1$, the symbol $I_{k}$ denote the sequence $(p-q+1,1, \ldots, 1,0)$ with $k$ non-zero entries. Then Morin singularities are singularities with symbols $I_{k}$. A Morin mapping is an $I_{k}$-mapping if it has no singularities of type $I_{k+1}$. For $k=1,2$ and 3 , points with the symbols $I_{k}$ are called fold, cusp and swallowtail singular points respectively. In this terminology, for example, a fold mapping is a mapping which has only fold singular points.

Given two manifolds $P$ and $Q$, we are interested in finding a mapping $P \rightarrow Q$ that has as simple singularities as possible. Let $f: P \rightarrow Q$ be an arbitrary general position mapping. For every symbol $I$, the $\mathbb{Z}_{2}$-homology class represented by the closure $\overline{\Sigma^{I}(f)}$ does not change under general position homotopy. Therefore the homology class $\left[\overline{\Sigma^{I}(f)}\right]$ gives an obstruction to elimination of $I$-singularities by homotopy.

In [5] Chess showed that if $p-q$ is odd and $k \geq 4$, then the homology obstruction corresponding to $I_{k}$-singularities vanishes. Chess conjectured that in this case every Morin mapping $f$ is homotopic to a mapping without $I_{k}$-singular points.

We will show that the statement of the Chess conjecture holds. Furthermore we will prove a stronger assertion.

Theorem 1.1 Let $P$ and $Q$ be two orientable manifolds, $p-q$ odd. Then the homotopy class of an arbitrary Morin mapping $f: P \rightarrow Q$ contains a cusp mapping.

Remark The standard complex projective plane $\mathbb{C} P^{2}$ does not admit a fold mapping [9] (see also [1], [12]). This shows that the homotopy class of $f$ may contain no mappings with only $I_{1}$-singularities.

Remark The assumption on the parity of the number $p-q$ is essential since in the case where $p-q$ is even homology obstructions may be nontrivial [5].

Remark We refer to an excellent review [11] for further comments. In particular, see Remark 4.6, where the authors indicate that Theorem 1.1 does not hold for non-orientable manifolds.

In [10] (see also [7]) Saeki and Sakuma describe a remarkable relation between the problem of the existence of certain Morin mappings and the Hopf invariant 
one problem. Using this relation the authors show that if the Euler characteristic of $P$ is odd, $Q$ is almost parallelizable, and there exists a cusp mapping $f: P \rightarrow Q$, then the dimension of $Q$ is $1,2,3,4,7$ or 8 .

Note that if the Euler characteristic of $P$ is odd, then the dimension of $P$ is even. We obtain the following corollary.

Corollary 1.2 Suppose the Euler characteristic of $P$ is odd and the dimension of an almost parallelizable manifold $Q$ is odd and different from 1,3,7. Then there exist no Morin mappings from $P$ into $Q$.

\section{Jet bundles and suspension bundles}

Let $P$ and $Q$ be two smooth manifolds of dimensions $p$ and $q$ respectively. $A$ germ at a point $x \in P$ is a mapping from some neighborhood about $x$ in $P$ into $Q$. Two germs are equivalent if they coincide on some neighborhood of $x$. The class of equivalence of germs (or simply the germ) at $x$ represented by a mapping $f$ is denoted by $[f]_{x}$.

Let $U$ be a neighborhood of $x$ in $P$ and $V$ be a neighborhood of $y=f(x)$ in $Q$. Let

$$
\tau_{U}:(U, x) \rightarrow\left(\mathbb{R}^{p}, 0\right) \quad \text { and } \quad \tau_{V}:(V, y) \rightarrow\left(\mathbb{R}^{q}, 0\right)
$$

be coordinate systems. Two germs $[f]_{x}$ and $[g]_{x}$ are $k$-equivalent if the mappings $\tau_{V} \circ f \circ \tau_{U}^{-1}$ and $\tau_{V} \circ g \circ \tau_{U}^{-1}$, which are defined in a neighborhood of $0 \in \mathbb{R}^{p}$, have the same derivatives at $0 \in \mathbb{R}^{p}$ of order $\leq k$. The notion of $k$ equivalence is well-defined, i.e. it does not depend on choice of representatives of germs and on choice of coordinate systems. A class of $k$-equivalent germs at $x$ is called $a k$-jet. The set of all $k$-jets constitute a set $J^{k}(P, Q)$. The projection $J^{k}(P, Q) \rightarrow P \times Q$ that takes a germ $[f]_{x}$ into a point $x \times f(x)$ turns $J^{k}(P, Q)$ into a bundle (for details see [4]), which is called the $k$-jet bundle over $P \times Q$.

Let $y$ be a point of a manifold and $V$ a neighborhood of $y$. We say that two functions on $V$ lead to the same local function at $y$, if at the point $y$ their partial derivatives agree. Thus a local function is an equivalence class of functions defined on a neighborhood of $y$. The set of all local functions at the point $y$ constitutes an algebra of jets $\mathcal{F}(y)$. Every smooth mapping $f:(U, x) \rightarrow(V, y)$ defines a homomorphism of algebras $f^{*}: \mathcal{F}(y) \rightarrow \mathcal{F}(x)$. The maximal ideal $m_{y}$ of $\mathcal{F}(y)$ maps under the homomorphism $f^{*}$ to the maximal ideal $m_{x} \subset \mathcal{F}(x)$. 
The restriction of $f^{*}$ to $m_{y}$ and the projection of $f^{*}\left(m_{y}\right) \subset m_{x}$ onto $m_{x} / m_{x}^{k+1}$ lead to a homomorphism

$$
f_{k, x}: m_{y} \rightarrow m_{x} / m_{x}^{k+1} .
$$

It is easy to verify that $k$-jets of mappings $(U, x) \rightarrow(V, y)$ are in bijective correspondence with algebra homomorphisms $m_{y} \rightarrow m_{x} / m_{x}^{k+1}$. That is why we will identify a $k$-jet with the corresponding homomorphism.

The projections of $P \times Q$ onto the factors induce from the tangent bundles TP and $T Q$ two vector bundles $\xi$ and $\eta$ over $P \times Q$. The latter bundles determine a bundle $\mathcal{H O M}(\xi, \eta)$ over $P \times Q$. The fiber of $\mathcal{H O M}(\xi, \eta)$ over a point $x \times y$ is the set of homomorphisms $\operatorname{Hom}\left(\xi_{x}, \eta_{y}\right)$ between the fibers of the bundles $\xi$ and $\eta$. The bundle $\xi$ determines the $k$-th symmetric tensor product bundle $\circ^{k} \xi$ over $P \times Q$, which together with $\eta$ leads to a bundle $\mathcal{H O M}\left({ }^{k} \xi, \eta\right)$.

Lemma 2.1 The $k$-jet bundle contains a vector subbundle $\mathcal{C}^{k}$ isomorphic to $\mathcal{H O M}\left(\circ^{k} \xi, \eta\right)$.

Proof Define $\mathcal{C}^{k}$ as the union of those $k$-jets $f_{k, x}$ which take $m_{y}$ to $m_{x}^{k}$. With each $f_{k, x} \in C^{k}$ we associate a homomorphism (for details, see [4, Theorem 4.1])

$$
\underbrace{\xi_{x} \circ \ldots \circ \xi_{x}}_{k} \otimes m_{y} / m_{y}^{2} \rightarrow \mathbb{R}
$$

which sends $v_{1} \circ \ldots \circ v_{k} \otimes \alpha$ into the value of $v_{1} \circ \ldots \circ v_{k}$ at a function representing $f_{k, x}(\alpha)$. In view of the isomorphism $m_{y} / m_{y}^{2} \approx \operatorname{Hom}\left(\eta_{y}, \mathbb{R}\right)$, the homomorphism (1) is an element of $\operatorname{Hom}\left({ }^{k} \xi_{x}, \eta_{y}\right)$. It is easy to verify that the obtained correspondence $C^{k} \rightarrow \mathcal{H O M}\left({ }^{k} \xi_{x}, \eta_{y}\right)$ is an isomorphism of vector bundles.

Corollary 2.2 There is an isomorphism $J^{k-1}(P, Q) \oplus \mathcal{C}^{k} \approx J^{k}(P, Q)$.

Proof Though the sum of two algebra homomorphisms may not be an algebra homomorphism, the sum of a homomorphism $f_{k, x} \in J^{k}(P, Q)$ and a homomorphism $h \in \mathcal{C}^{k}$ is a well defined homomorphism of algebras $\left(f_{k, x}+h\right) \in J^{k}(P, Q)$. This defines an action of $\mathcal{C}^{k}$ on $J^{k}(P, Q)$. Two $k$-jets $\alpha$ and $\beta$ map under the canonical projection

$$
J^{k}(P, Q) \longrightarrow J^{k}(P, Q) / \mathcal{C}^{k}
$$

onto one point if and only if $\alpha$ and $\beta$ have the same $(k-1)$-jet. Therefore $J^{k}(P, Q) / \mathcal{C}^{k}$ is canonically isomorphic to $J^{k-1}(P, Q)$. 
Remark The isomorphism $J^{k-1}(P, Q) \oplus \mathcal{C}^{k} \approx J^{k}(P, Q)$ constructed in Corollary 2.2 is not canonical, since there is no canonical projection of the $k$-jet bundle onto $\mathcal{C}^{k}$.

In [8] Ronga introduced the bundle

$$
S^{k}(\xi, \eta)=\mathcal{H O M}(\xi, \eta) \oplus \mathcal{H O} \mathcal{M}(\xi \circ \xi, \eta) \oplus \ldots \oplus \mathcal{H O M}\left(\circ^{k} \xi, \eta\right),
$$

which we will call the $k$-suspension bundle over $P \times Q$.

Corollary 2.3 The $k$-jet bundle is isomorphic to the $k$-suspension bundle.

\section{Submanifolds of singularities}

There are canonical projections $J^{k+1}(P, Q) \rightarrow J^{k}(P, Q)$, which lead to the infinite dimensional jet bundle $J(P, Q):=\underline{\lim } J^{k}(P, Q)$. Let $f: P \rightarrow Q$ be a smooth mapping. Then at every point $x \times f(x)$ of the manifold $P \times Q$, the mapping $f$ determines a $k$-jet. The $k$-jets defined by $f$ lead to a mapping $j^{k} f$ of $P$ to the $k$-jet bundle. These mappings agree with projections of $\lim ^{k}(P, Q)$ and therefore define a mapping $j f: P \rightarrow J(P, Q)$, which is called the jet extension of $f$. We will call a subset of $J(P, Q)$ a submanifold of the jet bundle if it is the inverse image of a submanifold of some $k$-jet bundle. A function $\Phi$ on the jet bundle is said to be smooth if locally $\Phi$ is the composition of the projection onto some $k$-jet bundle and a smooth function on $J^{k}(P, Q)$. In particular, the composition $\Phi \circ j f$ of a smooth function $\Phi$ on $J(P, Q)$ and a jet extension $j f$ is smooth. A tangent to the jet bundle vector is a differential operator. A tangent to $J(P, Q)$ bundle is defined as a union of all vectors tangent to the jet bundle.

Suppose that at a point $x \in P$ the mapping $f$ determines a jet $z$. Then the differential of $j f$ sends differential operators at $x$ to differential operators at $z$, that is $d(j f)$ maps $T_{x} P$ into some space $D_{z}$ tangent to the jet bundle. In fact, the space $D_{z}$ and the isomorphism $T_{x} P \rightarrow D_{z}$ do not depend on representative $f$ of the jet $z$. Let $\pi$ denote the composition of the jet bundle projection and the projection of $P \times Q$ onto the first factor. Then the tangent bundle of the jet space contains a subbundle $D$, called the total tangent bundle, which can be identified with the induced bundle $\pi^{*} T P$ by the property: for any vector field $v$ on an open set $U$ of $P$, any jet extension $j f$ and any smooth function $\Phi$ on $J(P, Q)$, the section $V$ of $D$ over $\pi^{-1}(U)$ corresponding to $v$ satisfies the equation

$$
V \Phi \circ j f=v(\Phi \circ j f) .
$$


We recall that the projections $P \times Q$ onto the factors induce two vector bundles $\xi$ and $\eta$ over $P \times Q$ which determine a bundle $\mathcal{H O M}(\xi, \eta)$. There is a canonical isomorphism between the 1 -jet bundle and the bundle $\mathcal{H O} \mathcal{M}(\xi, \eta)$. Consequently 1 -jet component of a $k$-jet $z$ at a point $x \in P$ defines a homomorphism $h: T_{x} P \rightarrow T_{y} Q, y=z(x)$. We denote the kernel of the homomorphism $h$ by $K_{1, z}$. Identifying the space $T_{x} P$ with the fiber $D_{z}$ of $D$, we may assume that $K_{1, z}$ is a subspace of $D_{z}$. Hence at every point $z \in J(P, Q)$ we have a space $K_{1, z}$. Boardman showed that the union $\Sigma^{i}=\Sigma^{i}(P, Q)$ of jets $z$ with $\operatorname{dim} K_{1, z}=i$ is a submanifold of $J(P, Q)$.

Suppose that we have already defined a submanifold $\Sigma_{n-1}=\Sigma^{i_{1}, \ldots, i_{n-1}}$ of the jet space. Suppose also that at every point $z \in \Sigma_{n-1}$ we have already defined a space $K_{n-1, z}$. Then the space $K_{n, z}$ is defined as $K_{n-1, z} \cap T_{z} \Sigma_{n-1}$ and $\Sigma_{n}$ is defined as the set of points $z \in \Sigma_{n-1}$ such that $\operatorname{dim} K_{n, z}=i_{n}$. Boardman proved that the sets $\Sigma_{n}$ are submanifolds of $J(P, Q)$. In particular every submanifold $\Sigma_{n}$ comes from a submanifold of an appropriate finite dimensional $k$-jet space. In fact the submanifold with symbol $I_{n}$ is the inverse image of the projection of the jet space onto $n$-jet bundle. To simplify notation, we denote the projections of $\Sigma_{n}$ to the $k$-jet bundles with $k \geq n$ by the same symbol $\Sigma_{n}$.

Let us now turn to the $k$-suspension bundle. Following the paper [4], we will define submanifolds $\tilde{\Sigma}^{I}$ of the $k$-suspension bundle.

A point of the $k$-suspension bundle over a point $x \times y \in P \times Q$ is the set of homomorphisms $h=\left(h_{1}, \ldots, h_{k}\right)$, where $h_{i} \in \operatorname{Hom}\left(\circ^{i} \xi_{x}, \eta_{y}\right)$. For every $k$ suspension $h$ we will define a sequence of subspaces $T_{x} P=K_{0} \supset K_{1} \supset \ldots \supset K_{k}$. Then we will define the singular set $\tilde{\Sigma}^{i_{1}, \ldots, i_{n}}$ as

$$
\tilde{\Sigma}^{i_{1}, \ldots, i_{n}}=\left\{h \mid \operatorname{dim} K_{j}=i_{j} \text { for } j=1, \ldots, n\right\} .
$$

We start with definition of a space $K_{1} \supset K_{0}$ and a projection of $P_{0}=T_{y} Q$ onto a factor space $Q_{1}$. The $h_{1}$-component of $h$ is a homomorphism of $K_{0}$ into $P_{0}$. We define $K_{1}$ and $Q_{1}$ as the kernel and the cokernel of $h_{1}$ :

$$
0 \longrightarrow K_{1} \longrightarrow K_{0} \stackrel{h_{1}}{\longrightarrow} P_{0} \longrightarrow Q_{1} \longrightarrow 0 .
$$

The cokernel homomorphism of this exact sequence gives rise to a homomorphism $\operatorname{Hom}\left(K_{1}, P_{0}\right) \rightarrow \operatorname{Hom}\left(K_{1}, Q_{1}\right)$, coimage of which is denoted by $P_{1}$. The sequence of the homomorphisms

$$
\operatorname{Hom}\left(K_{1} \circ K_{1}, P_{0}\right) \rightarrow \operatorname{Hom}\left(K_{1}, \operatorname{Hom}\left(K_{1}, P_{0}\right)\right) \rightarrow \operatorname{Hom}\left(K_{1}, P_{1}\right)
$$

takes the restriction of $h_{2}$ on $K_{1} \circ K_{1}$ to a homomorphism $\sigma\left(h_{2}\right): K_{1} \rightarrow P_{1}$. Again the spaces $K_{2}$ and $Q_{2}$ are respectively defined as the kernel and the cokernel of the homomorphism $\sigma\left(h_{2}\right)$. 
The definition continues by induction. In the $n$-th step we are given some spaces $K_{i}, Q_{i}$ for $i \leq n$, spaces $P_{i}$ for $i \leq n-1$ and projections

$$
\begin{gathered}
\operatorname{Hom}\left(K^{n-1}, P_{0}\right) \rightarrow P_{n-1}, \\
P_{n-1} \rightarrow Q_{n},
\end{gathered}
$$

where $K^{n-1}$ abbreviates the product $K_{n-1} \circ \ldots \circ K_{1}$.

First we define $P_{n}$ as the coimage of the composition

$$
\operatorname{Hom}\left(K^{n}, P_{0}\right) \rightarrow \operatorname{Hom}\left(K_{n}, \operatorname{Hom}\left(K^{n-1}, P_{0}\right)\right) \rightarrow \operatorname{Hom}\left(K_{n}, Q_{n}\right),
$$

where the latter homomorphism is determined by the two given projections. Then we transfer the restriction of the homomorphism $h_{n+1}$ on $K_{n} \circ K^{n}$ to a homomorphism $\sigma\left(h_{n+1}\right): K_{n} \rightarrow P_{n}$ using the composition

$$
\operatorname{Hom}\left(K_{n} \circ K^{n}, P_{0}\right) \rightarrow \operatorname{Hom}\left(K_{n}, \operatorname{Hom}\left(K^{n}, P_{0}\right)\right) \rightarrow \operatorname{Hom}\left(K_{n}, P_{n}\right) .
$$

Finally we define $K_{n+1}$ and $Q_{n+1}$ by the exact sequence

$$
0 \longrightarrow K_{n+1} \longrightarrow K_{n} \stackrel{\sigma\left(h_{n+1}\right)}{\longrightarrow} P_{n} \longrightarrow Q_{n+1} \longrightarrow 0 .
$$

In the previous section we established a homeomorphism between the fibers of the $k$-jet bundle and $k$-suspension bundle. Suppose that neighborhoods of points $x \in P$ and $y \in Q$ are equipped with coordinate systems. Then every $k$-jet $g$ which takes $x$ to $y$ has the canonical decomposition into the sum of $k$ jets $g_{i}, i=1, \ldots, k$, such that in the selected coordinates the partial derivatives of the jet $g_{i}$ at $x$ of order $\neq i$ and $\leq k$ are trivial. In other words the choice of local coordinates determines a homeomorphism

$$
\left.\left.\left.J^{k}(P, Q)\right|_{x \times y} \rightarrow \mathcal{C}^{1}\right|_{x \times y} \oplus \ldots \oplus \mathcal{C}^{k}\right|_{x \times y} .
$$

Since $\left.\mathcal{C}^{i}\right|_{x \times y}$ is isomorphic to $\operatorname{Hom}\left(\circ^{i} \xi_{x}, \eta_{y}\right)$, we obtain a homeomorphism between the fibers of the $k$-jet bundle and $k$-suspension bundle.

Remark From [4] we deduce that this homeomorphism takes the singular submanifolds $\Sigma^{I}$ to $\tilde{\Sigma}^{I}$. Suppose that a $k$-jet $z$ maps onto a $k$-suspension $h=\left(h_{1}, \ldots, h_{k}\right)$. The homomorphisms $\left\{h_{i}\right\}$ depends not only on $z$ but also on choice of coordinates in $U_{i}$. However Boardman [4] showed that the spaces $K_{i}$, $Q_{i}, P_{i}$ and the homomorphisms $\sigma\left(h_{i}\right)$ defined by $h$ are independent from the choice of coordinates.

Lemma 3.1 For every integer $k \geq 1$, there is a homeomorphism of bundles $r_{k}: J^{k}(P, Q) \rightarrow S^{k}(\xi, \eta)$ which takes the singular sets $\Sigma^{I}$ to $\tilde{\Sigma}^{I}$. 
Proof Choose covers of $P$ and $Q$ by closed discs. Let $U_{1}, \ldots, U_{t}$ be the closed discs of the product cover of $P \times Q$. For each disc $U_{i}$, choose a coordinate system which comes from some coordinate systems of the two disc factors of $U_{i}$. We will write $J^{k}$ for the $k$-jet bundle and $\left.J^{k}\right|_{U_{i}}$ for its restriction on $U_{i}$. We adopt similar notations for the $k$-suspension bundle. The choice of coordinates in $U_{i}$ leads to a homeomorphism

$$
\beta_{i}:\left.\left.J^{k}\right|_{U_{i}} \rightarrow S^{k}\right|_{U_{i}}
$$

Let $\left\{\varphi_{i}\right\}$ be a partition of unity for the cover $\left\{U_{i}\right\}$ of $P \times Q$. We define $r_{k}: J^{k} \rightarrow S^{k}$ by

$$
r_{k}=\varphi_{1} \beta_{1}+\varphi_{2} \beta_{2}+\ldots+\varphi_{k} \beta_{k} .
$$

Suppose that $U_{i} \cap U_{j}$ is nonempty and $z$ is a $k$-jet at a point of $U_{i} \cap U_{j}$. Suppose

$$
\beta_{i}(z)=\left(h_{1}^{i}, \ldots, h_{k}^{i}\right) \text { and } \beta_{j}(z)=\left(h_{1}^{j}, \ldots, h_{k}^{j}\right) .
$$

Then by the remark preceding the lemma, the homomorphisms $\sigma\left(h_{s}^{i}\right)$ and $\sigma\left(h_{s}^{j}\right)$ coincide for all $s=1, \ldots, k$. Consequently, $r_{k}$ takes $\Sigma^{I}$ to $\tilde{\Sigma}^{I}$.

The mapping $r_{k}$ is continuous and open. Hence to prove that $r_{k}$ is a homeomorphism it suffices to show that $r_{k}$ is one-to-one.

For $k=1$, the mapping $r_{k}$ is the canonical isomorphism. Suppose that $r_{k-1}$ is one-to-one and for some different $k$-jets $z_{1}$ and $z_{2}$, we have $r_{k}\left(z_{1}\right)=r_{k}\left(z_{2}\right)$. Since $r_{k-1}$ is one-to-one, the $k$-jets $z_{1}$ and $z_{2}$ have the same $(k-1)$-jet components. Hence there is $v \in \mathcal{C}^{k}$ for which $z_{1}=z_{2}+v$. Here we invoke the fact that $\mathcal{C}^{k}$ has a canonical action on $J^{k}$.

For every $i$, we have $\beta_{i}\left(z_{1}\right)=\beta_{i}\left(z_{2}\right)+\beta_{i}(v)$. Therefore

$$
r_{k}\left(z_{1}\right)=r_{k}\left(z_{2}\right)+r_{k}(v) .
$$

The restriction of the mapping $r_{k}$ to $\mathcal{C}^{k}$ is a canonical identification of $\mathcal{C}^{k}$ with $\mathcal{H O M}\left(\circ^{k} \xi_{k}, \eta\right)$. Hence $r_{k}(v) \neq 0$. Then (3) implies that $r_{k}\left(z_{1}\right) \neq r_{k}\left(z_{2}\right)$.

Corollary 3.2 There is an isomorphism of bundles $r: J(P, Q) \rightarrow S(\xi, \eta)$ which takes every set $\Sigma_{n}$ isomorphically onto $\tilde{\Sigma}_{n}$.

The space $J^{k}(P, Q)$ may be also viewed as a bundle over $P$ with projection

$$
\pi: J^{k}(P, Q) \rightarrow P \times Q \rightarrow P .
$$

Let $f: P \rightarrow Q$ be a smooth mapping. Then at every point $p \in P$ the mapping $f$ defines a $k$-jet. Consequently, every mapping $f: P \rightarrow Q$ gives rise to a section $j^{k} f: P \rightarrow J^{k}(P, Q)$, which is called the $k$-extension of $f$ or the $k$-jet 
section afforded by $f$. The sections $\left\{j^{k} f\right\}_{k}$ determined by a smooth mapping $f$ commute with the canonical projections $J^{k+1}(P, Q) \rightarrow J^{k}(P, Q)$. Therefore every smooth mapping $f: P \rightarrow Q$ also defines a section $j f: P \rightarrow J(P, Q)$, which is called the jet extension of $f$.

A smooth mapping $f$ is in general position if its jet extension is transversal to every singular submanifold $\Sigma^{I}$. By the Thom Theorem every mapping has a general position approximation.

Let $f$ be a general position mapping. Then the subsets $(j f)^{-1}\left(\Sigma^{I}\right)$ are submanifolds of $P$. Every condition $k r_{x}\left(f_{n-1}\right)=i_{n}$ in the definition of $\Sigma^{I}(f)$ can be substituted by the equivalent condition $\operatorname{dim} K_{n, x}(f)=i_{n}$, where the space $K_{n, x}(f)$ is the intersection of the kernel of $d f$ at $x$ and the tangent space $T_{x} \Sigma_{n-1}(f)$. Hence the sets $(j f)^{-1}\left(\Sigma^{I}\right)$ coincide with the sets $\Sigma^{I}(f)$. In particular the jet extension of a mapping $f$ without $I$-singularities does not intersect the set $\Sigma^{I}$.

Let $\Omega_{r}=\Omega_{r}(P, Q) \subset J(P, Q)$ denote the union of the regular points and the Morin singular points with indexes of length at most $r$.

Theorem 3.3 (Ando-Eliashberg, [2], [6]) Let $f: P^{p} \rightarrow Q^{q}, p \geq q \geq 2$, be a continuous mapping. The homotopy class of the mapping $f$ contains an $I_{r}$-mapping, $r \geq 1$, if and only if there is a section of the bundle $\Omega_{r}$.

Note that every general position mapping $f: P^{p} \rightarrow Q^{q}, q=1$, is a fold mapping. That is why for $q=1$, Theorem 1.1 holds and we will assume that $q \geq 2$.

Let $\tilde{\Omega}_{r}$ denote the subset of the suspension bundle corresponding to the set $\Omega_{r}(P, Q) \subset J(P, Q)$. Every mapping $f: P \rightarrow Q$ defines a section $j f$ of $J(P, Q)$. The composition $r \circ(j f)$ is a section of $S(P, Q)$. In view of Lemma 3.1 the Ando-Eliashberg Theorem implies that to prove that the homotopy class of a mapping $f$ contains a cusp mapping, it suffices to show that the section of the suspension bundle defined by $f$ is homotopic to a section of the bundle $\tilde{\Omega}_{2} \subset S(\xi, \eta)$.

\section{Proof of Theorem 1.1}

We recall that in a neighborhood of a fold singular point $x$, the mapping $f$ has the form

$$
\begin{aligned}
T_{i} & =t_{i}, \quad i=1,2, \ldots, q-1 \\
Z & =Q(x), \quad Q(x)= \pm k_{1}^{2} \pm \ldots \pm k_{p-q+1}^{2} .
\end{aligned}
$$


If $x$ is an $I_{r}$-singular point of $f$ and $r>1$, then in some neighborhood about $x$ the mapping $f$ has the form

$$
\begin{aligned}
T_{i} & =t_{i}, \quad i=1,2, \ldots, q-r, \\
L_{i} & =l_{i}, \quad i=2,3, \ldots, r, \\
Z & =Q(x)+\sum_{t=2}^{r} l_{t} k^{t-1}+k^{r+1}, \quad Q(x)= \pm k_{1}^{2} \pm \ldots \pm k_{p-q}^{2} .
\end{aligned}
$$

Let $f: P \rightarrow Q$ be a Morin mapping, for which the set $\Sigma_{2}(f)$ is nonempty. We define the section $f_{i}: P \rightarrow \operatorname{Hom}\left(\circ^{i} \xi, \eta\right)$ as the $i$-th component of the section $r \circ(j f)$ of the suspension bundle $S(\xi, \eta) \rightarrow P$. Over $\overline{\Sigma_{2}(f)}$ the components $f_{1}$ and $f_{2}$ defined by the mapping $f$ determine the bundles $K_{i}, Q_{i}, i=1,2$ and the exact sequences

$$
\begin{gathered}
0 \longrightarrow K_{1} \longrightarrow T P \longrightarrow T Q \longrightarrow Q_{1} \longrightarrow 0, \\
0 \longrightarrow K_{2} \longrightarrow K_{1} \longrightarrow \mathcal{H O M}\left(K_{1}, Q_{1}\right) \longrightarrow Q_{2} \longrightarrow 0 .
\end{gathered}
$$

From the latter sequence one can deduce that the bundle $Q_{2}$ is canonically isomorphic to $\mathcal{H O} \mathcal{M}\left(K_{2}, Q_{1}\right)$ and that the homomorphism

$$
K_{1} / K_{2} \otimes K_{1} / K_{2} \longrightarrow Q_{1},
$$

which is defined by the middle homomorphism of the second exact sequence, is a non-degenerate quadratic form (see Chess, [5]). Since the dimension of $K_{1} / K_{2}$ is odd, the quadratic form (6) determines a canonical orientation of the bundle $Q_{1}$. In particular the 1-dimensional bundle $Q_{1}$ is trivial. This observation also belongs to Chess [5].

Assume that the bundle $K_{2}$ is trivial. Then the bundle $Q_{2}$ being isomorphic to $\mathcal{H O M}\left(K_{2}, Q_{1}\right)$ is trivial as well. Let

$$
\tilde{h}: K_{2} \rightarrow \mathcal{H O M}\left(K_{2}, Q_{2}\right) \approx \mathcal{H O M}\left(K_{2} \otimes K_{2}, Q_{1}\right)
$$

be an isomorphism over $\overline{\Sigma_{2}(f)}$ and $h: P \rightarrow \mathcal{H O M}\left(\circ^{3} \xi, \eta\right)$ an arbitrary section, the restriction of which on $0^{3} K_{2}$ over $\overline{\Sigma_{2}(f)}$ followed by the projection given by $\eta \rightarrow Q_{1}$, induces the homomorphism $\tilde{h}$. Then the section of a suspension bundle whose first three components are $f_{1}, f_{2}$ and $h$ is a section of the bundle $\tilde{\Omega}_{2}$. Since for $i>0$ the bundle $\mathcal{H O M}\left(\circ^{i} \xi, \eta\right)$ is a vector bundle, we have that the composition $r \circ(j f)$ is homotopic to the section $s$ and therefore the original mapping $f$ is homotopic to a cusp mapping.

Now let us prove the assumption that $K_{2}$ is trivial over $\overline{\Sigma_{2}(f)}$.

Lemma 4.1 The submanifold $\overline{\Sigma_{2}(f)}$ is canonically cooriented in the submanifold $\overline{\Sigma_{1}(f)}$. 
Proof For non-degenerate quadratic forms of order $n$, we adopt the convention to identify the index $\lambda$ with the index $n-\lambda$. Then the index ind $Q(x)$ of the quadratic form $Q(x)$ in (4) and (5) does not depend on choice of coordinates.

With every $I_{k}$-singular point $x$ by (4) and (5) we associate a quadratic mapping of the form $Q(x)$. It is easily verified that for every cusp singular point $y$ and a fold singular point $x$ of a small neighborhood of $y$, we have $Q(x)=$ $Q(y) \pm k_{p-q+1}^{2}$. Moreover, if $x_{1}$ and $x_{2}$ are two fold singular points and there is a path joining $x_{1}$ with $x_{2}$ which intersects $\overline{\Sigma_{2}(f)}$ transversally and at exactly one point, then ind $Q\left(x_{1}\right)$ - ind $Q\left(x_{2}\right)= \pm 1$. In particular, the normal bundle of $\overline{\Sigma_{2}(f)}$ in $\overline{\Sigma_{1}(f)}$ has a canonical orientation.

Lemma 4.2 Over every connected component of $\Sigma_{2}(f)$ the bundle $K_{2}$ has a canonical orientation.

Proof At every point $x \in \overline{\Sigma_{2}(f)}$ there is an exact sequence

$$
0 \longrightarrow K_{3, x} \longrightarrow K_{2, x} \longrightarrow \mathcal{H O M}\left(K_{2, x}, Q_{2, x}\right) \longrightarrow Q_{3, x} \longrightarrow 0 .
$$

If the point $x$ is in fact a cusp singular point, then the space $K_{3, x}$ is trivial and therefore the sequence reduces to

$$
0 \longrightarrow K_{2, x} \longrightarrow \mathcal{H O} \mathcal{M}\left(K_{2, x}, Q_{2, x}\right) \longrightarrow 0
$$

and gives rise to a quadratic form

$$
K_{2, x} \otimes K_{2, x} \longrightarrow Q_{2, x} \approx \mathcal{H O} \mathcal{M}\left(K_{2, x}, Q_{1, x}\right) .
$$

This form being non-degenerate orients the space $\mathcal{H O M}\left(K_{2, x}, Q_{1, x}\right)$. Since $Q_{1, x}$ has a canonical orientation, we obtain a canonical orientation of $K_{2, x}$.

Let $\gamma:[-1,1] \rightarrow \overline{\Sigma_{2}(f)}$ be a path which intersects the submanifold of non-cusp singular points transversally and at exactly one point.

Lemma 4.3 The canonical orientations of $K_{2}$ at $\gamma(-1)$ and $\gamma(1)$ lead to different orientations of the trivial bundle $\gamma^{*} K_{2}$.

Proof If necessary we slightly modify the path $\gamma$ so that the unique intersection point of $\gamma$ and the set $\overline{\Sigma_{3}(f)}$ is a swallowtail singular point. Then the statement of the lemma is easily verified using the formulas (5).

Now we are in position to prove the assumption. 
Lemma 4.4 The bundle $K_{2}$ is trivial over $\overline{\Sigma_{2}(f)}$.

Proof Assume that the statement of the lemma is wrong. Then there is a closed path $\gamma: S^{1} \rightarrow \overline{\Sigma_{2}(f)}$ which induces a non-orientable bundle $\gamma^{*} K_{2}$ over the circle $S^{1}$.

We may assume that the path $\gamma$ intersects the submanifold $\overline{\Sigma_{3}(f)}$ transversally. Let $t_{1}, \ldots, t_{k}, t_{k+1}=t_{1}$ be the points of the intersection $\gamma \cap \overline{\Sigma_{3}(f)}$. Over every interval $\left(t_{i}, t_{i+1}\right)$ the normal bundle of $\overline{\Sigma_{2}(f)}$ in $\overline{\Sigma_{1}(f)}$ has two orientations. One orientation is given by Lemma 4.1 and another is given by the canonical orientation of the bundle $K_{2}$. By Lemma 4.3 if these orientations coincide over $\left(t_{i-1}, t_{i}\right)$, then they differ over $\left(t_{i}, t_{i+1}\right)$. Therefore the number of the intersection points is even and the bundle $\gamma^{*} K_{2}$ is trivial. Contradiction.

Remark The statement similar to the assertion of Lemma 4.4 for the jet bundle $J(P, Q)$ is not correct. The vector bundle $K_{2}$ over $\overline{\Sigma^{I_{2}}} \subset J(P, Q)$ is nonorientable. This follows for example from the study of topological properties of $\Sigma^{I_{r}}$ in $[2, \S 4]$.

\section{References}

[1] P. Akhmetev, R. Sadykov, A remark on elimination of singularities for mappings of 4-manifold into 3-manifold, Top. Appl., 131 (2003), 51-55.

[2] Y. Ando, On the elimination of Morin singularities, J. Math. Soc. Japan, 37 (1985), 471-487; Erratum 39 (1987), 537.

[3] V. I. Arnol'd, V. A. Vasil'ev, V. V. Goryunov, O. V. Lyashenko, Dynamical systems VI. Singularities, local and global theory, Encyclopedia of Mathematical Sciences - Vol. 6 (Springer, Berlin, 1993).

[4] J. M. Boardman, Singularities of differentiable maps, Publ. Math., 33 (1967), 21-57.

[5] D. S. Chess, $A$ note on the classes $\left[S_{1}^{k}(f)\right]$, Proc. Symp. Pure Math., 40 (1983), 221-224.

[6] J. M. Eliashberg, Surgery of singularities of smooth mappings, Math. USSR Izv., 6 (1972), 1302-1326.

[7] S. Kikuchi, O. Saeki, Remarks on the topology of folds, Proc. Amer. Math. Soc., 123 (1995), 905-908.

[8] F. Ronga, Le calculus des classes duales singularités de Boardman d'ordre deux, Comment. Math. Helv., 47 (1972), 15-35.

[9] O. Saeki, Notes on the topology of folds, J. Math. Soc. Japan, v.44, 3 (1992), 551-566. 
[10] O. Saeki, K. Sakuma, Maps with only Morin singularities and the Hopf invariant one problem, Math. Proc. Camb. Phil. Soc., 124 (1998), 501-511.

[11] O. Saeki, K. Sakuma, Elimination of Singularities: Thom Polynomials and Beyond, London Math. Soc., Lecture Notes Ser. 263.

[12] K. Sakuma, A note on nonremovable cusp singularities, Hiroshima Math. J., 31 (2001), 461-465.

University of Florida, Department of Mathematics,

358 Little Hall, 118105, Gainesville, Fl, 32611-8105, USA

Email: sadykov@math.ufl.edu

Received: 18 February 2003 Revised: 23 July 2003 